\title{
Acoustic coupling between two air bubbles in water
}

\author{
Pai-Yi HSIAO \\ Laboratoire de Physique Théorique de la Matière Condensée \\ Université Paris 7 - Denis Diderot \\ case 7020, 2 place jussieu, 75251 Paris Cedex 05, FRANCE \\ E-mail: hsiao@ccr.jussieu.fr \\ Martin DEVAUD and Jean-Claude BACRI \\ Laboratoire des Milieux Désordonnés et Hétérogènes \\ Université Pierre et Marie Curie - Paris 6 \\ case 78, 4 place jussieu, 75252 Paris Cedex 05, FRANCE \\ E-mails: devaud@ccr.jussieu.fr,jcbac@ccr.jussieu.fr
}

\begin{abstract}
Abstract - The acoustic coupling between two air bubbles immersed in water is clearly demonstrated. The system is acoustically forced, and its response is detected. The experimental results confirm that both theoretically predicted eigenmodes, respectively symmetrical and antisymmetrical, do exist. Their frequencies, measured as a function of the bubbles spacing, follow theoretical estimations within a $10 \%$ accuracy.
\end{abstract}

Keywords: bubbles, eigenmodes, acoustics

PACS : 43.20+g, 43.30Jx, 43.25Yw

Typeset using REVTEX 


\section{INTRODUCTION}

Bubbles play an important role in the sound propagation in everyday life liquids. For example, the murmur of the brooks essentially originates, as first suggested by Bragg [1],2], in the oscillations of air bubbles captured and dragged along by the water. The so-called "hot chocolate effect", namely the rising of sound pitch when one repeatedly taps the bottom of the mug in which some instant coffee or chocolate is being dissolved, is explained by the releasing into the water of the tiny bubbles trapped in the powder [3, [4]. Bubble dynamics and acoustic properties of liquids containing a large number of bubbles have been widely studied for a long time [5-10]. A very good and complete review in this domain was achieved by Leighton [11]. Inter alia, the problem of the interaction of two neighbouring bubbles has been discussed using fluid dynamics tools [12] or the acoustic-electrostatic analogy [13. Moreover, the free oscillations of a system of two (and even three) air cavities formed in a metal plate lying on a water surface have been theoretically and experimentally investigated

in detail (including cubic nonlinearities) [14. The aim of the present article is to present a simple, readily reproducible, experimental study of the forced oscillation regime of a two-air bubble system in water. We begin with a short introductory theory in which we show that the two-bubble system is mostly equivalent to a set of two magnetically coupled electric circuits.

\section{THEORETICAL MODEL}

An air bubble in water will be considered as a perfect sphere' of radius $R(t)=R_{0}+\xi(t)$, with variation $\xi$ much smaller than equilibrium value $R_{0}$. It can be shown that $\xi(t)$ oscillates

\footnotetext{
1 The correction to the Minnaert angular frequency due to deviation from the spherical shape can be shown to be negligible [15 17].
} 
with Minnaert's angular frequency $\omega_{0}=\sqrt{3 \gamma P_{0} / \rho_{0} R_{0}^{2}}$, where $\gamma$ is the specific heat ratio $C_{p} / C_{v}$ of air, and $P_{0}$ and $\rho_{0}$ respectively stand for the equilibrium pressure ${ }^{2}$ and mass density of water. This oscillation is damped through several mechanisms: of course the acoustic radiation damping (thanks to which the bubble noise is audible), but also the viscous and thermal dampings [5,7]. We will neglect, in the following simplified theory, the last two ones. Moreover, allowing for the typical $1 \mathrm{KHz}$ acoustic frequency and $1 \mathrm{~mm}$ bubble size we deal with in our experiment, we will neglect any sound propagation in the enclosed air. We thus deliberately restrict the present study to the (radial) fundamental resonance of the air bubble-water system.

\subsection{One-bubble free oscillation}

Let us consider one bubble with radius $R_{0}$ immersed in an infinite volume of water at equilibrium pressure $P_{0}$. Let $P(\vec{r}, t)$ be the actual pressure at site $\vec{r}$ and instant $t$. The extra pressure $p(\vec{r}, t)$ is defined as $P(\vec{r}, t)-P_{0}$. According to Minnaert's assumption, the enclosed air undergoes isentropic transformations and its (extra) pressure $p(t)$ is homogeneous inside the bubble. Then, neglecting air's inertia as well as the air-water surface tension, $p(t)$ and the radius variation $\xi(t)$ are linked by:

$$
\frac{p(t)}{P_{0}}+\frac{3 \gamma \xi(t)}{R_{0}}=0
$$

On the other hand, it can be easily shown that (extra) pressure $p(r, t)$ at distance $r$ from the center of the bubble follows a d'Alembert-like 1D equation, the solution of which exactly reads, for $r \geq R_{0}$ :

\footnotetext{
2 The pressure difference accross the bubble boundary due to air-water surface tension is about $1 \% P_{0}$ for a typical radius of $1 \mathrm{~mm}$ (see [5] eq. $(65 \mathrm{~b})$ ) and will be neglected: $P_{0}$ is also the equilibrium pressure of enclosed air.
} 


$$
p(r, t)=\frac{1}{r} \rho_{0} R_{0}^{2}\left[\xi^{\prime \prime}-\frac{R_{0}}{c} \xi^{\prime \prime \prime}+\ldots+\left(-\frac{R_{0}}{c}\right)^{k} \xi^{(2+k)}+\ldots\right]\left(t-\frac{r-R_{0}}{c}\right)
$$

where $c$ is the sound velocity in water. If the acoustic wavelength $\lambda$ is much larger than $r$ (i.e., under the circumstances, if condition $r \omega_{0} / c \ll 1$ is fulfilled), then $p(r, t)$ can be approximated by:

$$
\begin{aligned}
p(r, t) & \simeq \rho_{0} R_{0}^{2}\left[\frac{\xi^{\prime \prime}(t)}{r}-\frac{\xi^{\prime \prime \prime}(t)}{c}\right] \\
& \simeq \rho_{0} R_{0}^{2}\left[\frac{\xi^{\prime \prime}(t)}{r}+\frac{\omega_{0}^{2}}{c} \xi^{\prime}(t)\right]
\end{aligned}
$$

Then, equalling $p(t)$ in eq. (1) to $p\left(R_{0}, t\right)$ in eq. (3), one gets, all calculations carried out:

$$
\xi^{\prime \prime}+\frac{\omega_{0}^{2} R_{0}}{c} \xi^{\prime}+\frac{3 \gamma P_{0}}{\rho_{0} R_{0}^{2}} \xi=\xi^{\prime \prime}+\Gamma_{r a d} \xi^{\prime}+\omega_{0}^{2} \xi=0
$$

which is the well-known differential equation of a weakly damped 1D harmonic oscillator.

\subsection{Two-bubble free oscillation}

Let us now add a second bubble, with the same (equilibrium) radius $R_{0}$, at a distance $d$ apart from the first one. Let $\overrightarrow{r_{i}}(i=1,2)$ be the (equilibrium) position of the $i^{\text {th }}$ bubble center, $\xi_{i}(t)$ its radius variation, $p_{i}(t)$ the (inner) extra pressure of the enclosed air, and $p_{i}(\vec{r}, t)$ (resp. $\vec{u}_{i}(\vec{r}, t)$ the would-be (outer)extra pressure (resp. displacement with respect to equilibrium) at point $\vec{r}$ and instant $t$ in the water medium if bubble $i$ was alone. Then, allowing for the superposition principle for small displacements, we assume that overall water extra pressure and displacement respectively read:

$$
\begin{aligned}
& p(\vec{r}, t)=p_{1}(\vec{r}, t)+p_{2}(\vec{r}, t) \\
& \vec{u}(\vec{r}, t)=\vec{u}_{1}(\vec{r}, t)+\vec{u}_{2}(\vec{r}, t)
\end{aligned}
$$

\footnotetext{
${ }^{3}$ Ratio $\Gamma_{\text {rad }} / \omega_{0}=\omega_{0} R_{0} / c$ is actually assumed to be much smaller than unity, as a consequence of the the validity condition of eq. (3).
} 
with, of course, $p_{i}(t)$ and $\xi_{i}(t)$ still linked by eq.(1). On the surface of the first bubble: $r_{1}=\left|\vec{r}-\vec{r}_{1}\right|=R_{0}, r_{2}=\left|\vec{r}-\vec{r}_{2}\right| \simeq d$, and $p(\vec{r}, t)=p_{1}(t)$. A similar constraint is required on the surface of the second bubble, where $r_{1} \simeq d$ and $r_{2}=R_{0}$. If the bubble spacing $d$ is much smaller than $\lambda$ (i.e. $\omega_{0} d / c \ll 1$ ) then eq. (3) is available and we finally get the following pair of coupled motion equations:

$$
\begin{aligned}
& \xi_{1}^{\prime \prime}+\alpha \xi_{2}^{\prime \prime}+\Gamma_{r a d}\left(\xi_{1}^{\prime}+\xi_{2}^{\prime}\right)+\omega_{0}^{2} \xi_{1}=0 \\
& \alpha \xi_{1}^{\prime \prime}+\xi_{2}^{\prime \prime}+\Gamma_{r a d}\left(\xi_{1}^{\prime}+\xi_{2}^{\prime}\right)+\omega_{0}^{2} \xi_{2}=0
\end{aligned}
$$

where $\alpha=R_{0} / d(<0.5)$ can be regarded as a dimensionless coupling constant. Observe, by the way, that if double condition: $R_{0} \ll d \ll \lambda$ is fulfilled, eqs. (7) and (8) are available (with $\alpha \simeq 0$ ), and dynamic variables $\xi_{i}$ are still coupled by radiation damping, since the dissipation terms do not involve $\alpha$.

Defining symmetrical and antisymmetrical normal variables $\phi_{s}(t)$ and $\phi_{a}(t)$ as respectively the sum and the difference of $\xi_{1}(t)$ and $\xi_{2}(t)$, we get the uncoupled equations system:

$$
\begin{aligned}
(1+\alpha) \phi_{s}^{\prime \prime}+2 \Gamma_{r a d} \phi_{s}^{\prime}+\omega_{0}^{2} \phi_{s} & =0 \\
(1-\alpha) \phi_{a}^{\prime \prime}+\omega_{0}^{2} \phi_{a} & =0
\end{aligned}
$$

It is noteworthy that, as far as only radiation is concerned, the symmetrical mode's damping rate is twice the single-bubble's one, while the antisymmetrical mode is undamped.This feature is easily understood in terms of constructive (resp. destructive) interference between the acoustic waves radiated by each bubble, and parallels a well-known situation in the atomic physics domain (super- and sub- radiant quantum states of a couple of identical atoms interacting with each other through the E.M. field). From eqs. (9) and (10), it is clear that the symmetrical mode has the lower angular frequency $\omega_{s}=\omega_{0} / \sqrt{1+\alpha}$, and the

\footnotetext{
${ }^{4}$ In our experiments $\lambda$ is of order $1 \mathrm{~m}$, while $d$ ranges from 1 to $5 \mathrm{~cm}$.
} 
antisymmetrical mode the higher one $\omega_{a}=\omega_{0} / \sqrt{1-\alpha}$. Observe that, leaving apart the calculation of radiative damping, it is very easy to derive above expressions of $\omega_{s, a}$ using the following trick. Let us consider the water as an uncompressible fluid (i.e. $c \rightarrow \infty$ ). The water displacement due to bubble $i$ 's motion simply reads:

$$
\vec{u}_{i}(\vec{r}, t)=\xi_{i}(t) \frac{R_{0}^{2}}{r_{i}^{2}} \vec{e}_{r_{i}}
$$

with $\vec{e}_{r_{i}}=\left(\vec{r}-\vec{r}_{i}\right) /\left|\vec{r}-\vec{r}_{i}\right|=\left(\vec{r}-\vec{r}_{i}\right) / r_{i}$. Then, allowing for eq. (6), the overall water kinetic energy $T$ is:

$$
\begin{aligned}
T & =\frac{1}{2} \rho_{0} \int d^{3} r \quad\left(\frac{\partial \vec{u}}{\partial t}\right)^{2} \\
& =\frac{1}{2} M_{0}\left(\xi_{1}^{\prime 2}+\xi_{2}^{\prime 2}+2 \alpha \xi_{1}^{\prime} \xi_{1}^{\prime}\right)
\end{aligned}
$$

where $M_{0}=4 \pi R_{0}^{3} \rho_{0}$ is the effective mass of either bubble. On the other hand, the total potential energy $V$ associated with the isentropic compressibility of the enclosed air reads:

$$
V=\frac{1}{2} K\left(\xi_{1}^{2}+\xi_{2}^{2}\right)
$$

where $K=12 \pi \gamma R_{0} P_{0}$ is the effective stiffness of either bubble. The Lagrange equations derived from $L=T-V$ are:

$$
\begin{aligned}
& \xi_{1}^{\prime \prime}+\alpha \xi_{2}^{\prime \prime}+\omega_{0}^{2} \xi_{1}=0 \\
& \alpha \xi_{1}^{\prime \prime}+\xi_{2}^{\prime \prime}+\omega_{0}^{2} \xi_{2}=0
\end{aligned}
$$

which is exactly the $c \rightarrow \infty$ limit of eqs. (7) and (8). It is worth noticing that eqs. (12) through (15) are formally equivalent to those of a system of two $(L, C)$ electric circuits coupled by mutual induction with coefficient $\alpha L$. In this analogy, $M_{0}$ and $K$ respectively correspond to $L$ and $1 / C$, and the $\xi_{i}$ 's to the electric charges $q_{i}$ of either capacitor. 


\subsection{Forced oscillation}

Let us now suppose that the above studied two-bubble system is driven by an external acoustic source with an angular frequency $\omega$ near Minnaert's one, $\omega_{0}$. The phase difference of the driving pressures on both bubbles can therefore be neglected, since $\omega d / c \ll 1$. Let $p_{e i}(t)$ be the external pressure undergone by bubble $i$. Motion eqs. (7) and (8) are then completed in:

$$
\begin{aligned}
& \xi_{1}^{\prime \prime}+\alpha \xi_{2}^{\prime \prime}+\Gamma_{\text {rad }}\left(\xi_{1}^{\prime}+\xi_{2}^{\prime}\right)+\omega_{0}^{2} \xi_{1}=-\frac{p_{e 1}(t)}{\rho_{0} R_{0}} \\
& \alpha \xi_{1}^{\prime \prime}+\xi_{2}^{\prime \prime}+\Gamma_{r a d}\left(\xi_{1}^{\prime}+\xi_{2}^{\prime}\right)+\omega_{0}^{2} \xi_{2}=-\frac{p_{e 2}(t)}{\rho_{0} R_{0}}
\end{aligned}
$$

or equivalently:

$$
\begin{aligned}
\phi_{s}^{\prime \prime}+\frac{2 \Gamma_{r a d}}{1+\alpha} \phi_{s}^{\prime}+\omega_{s}^{2} \phi_{s} & =F_{e s}(t) \\
\phi_{a}^{\prime \prime}+\omega_{a}^{2} \phi_{a} & =F_{e a}(t)
\end{aligned}
$$

with $F_{e s}(t)=-\left(p_{e 1}(t)+p_{e 2}(t)\right) / \rho_{0} R_{0}(1+\alpha)$ and $F_{e a}(t)=-\left(p_{e 1}(t)-p_{e 2}(t)\right) / \rho_{0} R_{0}(1-\alpha)$.

Solving for $\phi_{s}$ and $\phi_{a}$ in above eqs. (18) and (19), one gets $\xi_{1}(t)$ and $\xi_{2}(t)$, and consequently (using eq. (3)) quantities $p_{1}(\vec{r}, t)$ and $p_{2}(\vec{r}, t)$ at any point $\vec{r}$ of the medium. At last, comparing external (applied) pressure $p_{e}(\vec{r}, t)$ with the actual overall extra pressure $p(\vec{r}, t)=p_{e}(\vec{r}, t)+p_{1}(\vec{r}, t)+p_{2}(\vec{r}, t)$, we can experimentally measure the two-bubble system's response as a function of $\omega$. In this respect (and provided that the excitation-detection geometry allows it), resonances are expected for $\omega=\omega_{s}$ and $\omega=\omega_{a}$.

\section{EXPERIMENTS}

Our aim is to demonstrate the existence of both above mentioned modes. From an experimental point of view, it turns out to be easier to implement a forced oscillation scheme than a free oscillation one. We therefore present the former hereafter. 


\subsection{Experimental setup}

A small net (see fig.1), made up with a gauze maintained with a wire, is designed to catch up an air bubble in water and to fix it at any desired position without appreciably modifying acoustic impedance and spherical symmetry. Two such devices are used for studying the two-bubble system. The external driving source is a speaker and extrapressure $p(\vec{r}, t)$ is measured with a small microphone. A function generator, to which the speaker is connected, produces a c.w. sinusoidal signal with a frequency slowly swept from $f_{\text {low }}$ to $f_{\text {high }}$. The signal delivered by the microphone is transmitted to a lock-in amplifier which compares it with the reference one (delivered by the function generator) and decomposes it into real and imaginary parts. Both parts can be seen on an oscilloscope and recorded with a computer (see fig.2).

In a preliminary set of experiments, without any bubble in the aquarium, the response of the microphone is calibrated for different speaker-microphone configurations. Two kinds of configurations are presented in figure 3. In figs. 3(a) and 3(b) the configuration is deliberately asymmetrical: the microphone is mainly susceptible to bubble 2's motion, while the speaker selectively drives bubble 2 (fig. $3(\mathrm{a})$ ) or bubble 1 (fig. $3(\mathrm{~b})$ ), so that $F_{\text {ea }}(t)$ is nonzero: both modes can be excited and the associated motions detected. In fig. 3(c), the speaker is placed far from the bubbles; then, not only the phases, but also the amplitudes of the external pressures $p_{1 e}(t)$ and $p_{2 e}(t)$ undergone on either bubble are appreciably equal. In such a symmetrical excitation configuration, $F_{e a}(t)=0$, so that the antisymmetrical mode remains unexcited. Observe, by the way, that since distances $r_{1}$ and $r_{2}$ between the bubbles and the microphone are equal, the latter would detect no contribution from the antisymmetrical mode even though it was excited (see eqs.(3) and (5): $r_{1}=r_{2}$ and $\xi_{1}=-\xi_{2}$ yields $p_{1}\left(r_{1}, t\right)+$ $\left.p\left(r_{2}, t\right)=0\right)$. 


\subsection{Results and discussion}

In figs. 4(a) and 4(b), the imaginary part Imp of the output signal from the lockin amplifier is displayed versus the speaker frequency $f$ for various values of the bubbles spacing $d$. Figures $4(\mathrm{a})$ and $4(\mathrm{~b})$ respectively correspond to configurations $3(\mathrm{a})$ and 3(b). Two resonances can be made out in fig 4(a) and (though at a lesser degree) in fig 4(b). Observe that the sign of the signal at the higher frequency resonance is changed from 4(a) to 4(b), while the lower frequency one remains unchanged. This is consistent with the latter signal being associated with the symmetrical mode's resonance $\left(\omega_{s}=\omega_{0} / \sqrt{1+\alpha}<\omega_{0}\right.$, and $F_{\text {es }}$ unchanged from configuration $3(\mathrm{a})$ to $3(\mathrm{~b})$ ), and the former one with the antisymmetrical mode's resonance $\left(\omega_{a}=\omega_{0} / \sqrt{1-\alpha}>\omega_{0}\right.$, and $F_{e a}$ changed into $-F_{e a}$ from configuration $3(\mathrm{a})$ to $3(\mathrm{~b}))$.

It is noteworthy that both resonances have appreciably the same width. This is in contradiction with simplified eqs.(9) and (10) (or (18) and (19)), in which only the radiation damping was considered. In fact, as mentioned in introduction, other kinds of damping should be taken into account: if viscous damping is absolutely negligible for such large bubbles, thermal damping is not (see fig.8 in [19]), and may be at the origin of the linewidth. Further discussion of this point is out of the scope of the present paper. In figure 5, we have plotted, for both symmetrical and antisymmetrical modes, and for $R_{0} \simeq 2 \mathrm{~mm}$, the inverse squared frequency $f^{-2}$ (multiplied by a factor of $10^{7}$ ) versus the inverse bubble spacing $d^{-1}$, in order to get a visual check of theoretical relations:

$$
\begin{aligned}
& \frac{1}{f_{s}^{2}}=\frac{1}{f_{0}^{2}}+\frac{R_{0}}{f_{0}^{2}} \cdot \frac{1}{d} \\
& \frac{1}{f_{a}^{2}}=\frac{1}{f_{0}^{2}}-\frac{R_{0}}{f_{0}^{2}} \cdot \frac{1}{d}
\end{aligned}
$$

Although experimental points are appreciably aligned, the measured slopes are about $40 \%$ below theoretical prediction, suggesting that coupling constant $\alpha$ has been overesti- 
mated. In fact, theoretical value $\alpha=R_{0} / d$ was derived in eq.(12) when integrating the water kinetic energy density $\frac{1}{2} \rho_{0}\left(\frac{\partial \vec{u}}{\partial t}\right)^{2}$ over the whole space urally lowered if some obstacle lies between the bubbles and consequently screens (part of) the water flow?. Now, this is exactly what happens in configurations $3(\mathrm{a})$ and $3(\mathrm{~b})$ : to be able to excite the antisymmetrical mode, we are compelled to insert the speaker between the two bubbles, thus bringing about the above screening effect. In order to check this interpretation, we performed the same experiment with configuration 3(c), and recorded, for the symmetrical branch of the linear fitting of fig. 5, a slope of about $90 \%$ of the theoretically predicted value.

At last, it should be noted that, when blocked from the top by the net, the bubble is, strictly speaking, no longer spherical. As mentioned in footnote 1, such a deviation from the spherical shape is (almost) of no consequence, and we have used the formulas derived above in section 2 with $R_{0}$ standing for the radius of the sphere of equivalent volume (i.e. the radius of the bubble before it is captured by the net). Nevertheless, this feature of our experimental setup raises the following difficulty: since, in course of motion, the fixed point of the bubble is no longer its centre (as implicitly assumed in the theoretical model) but its top, expression (11) of the water displacement is no longer correct; a dipolar term should be added to the spherical monopolar one. As a consequence, the kinetic energy $T$ derived in eq.(12) is modified too. More precisely, an exact calulation shows that $T$ should be multiplied by a factor of $7 / 6$ and the coupling constant $\alpha$ by a factor of $1+R_{0}^{2} /\left(4 d^{2}\right)$. These corrections lie within our experimental accuracy. We have consequently neglected

\footnotetext{
5 More precisely: over the whole space outside the two bubbles (the inner air's inertia being negligible). Nevertheless, it can be shown that the coefficient of coupling term $\xi_{1}^{\prime} \xi_{2}^{\prime}$ in integral (12) does not depend on the bubbles radius $R_{0}$.

${ }^{6}$ The effective mass $M_{0}$ is modified too, but at a lesser degree.
} 
them. In this respect, it may be noted that the gauze in our device does not act like a rigid wall because the water can flow through the meshes of the net. The situation is therefore different from that discused in other studies [15,18] considering the influence of the proximity of a rigid boundary on the Minnaert frequency.

\section{CONCLUSION}

As a conclusion, the acoustic inertial coupling between two air bubbles in water has been experimentally put in evidence. Theoretical analysis shows that the two-bubble system is formally equivalent to a set of two magnetically coupled $(L, C)$ electric circuits, with two eigenmodes, respectively symmetrical and antisymmetrical. Experimental measurements and theoretical predictions are in 10\% accuracy agreement.

\section{ACKNOWLEDGMENT}

We gratefully thank Professor F.Massias for enriching discussions about the correction to the Minnaert frequency in the case of a bubble fixed from the top by a wide-mesh net. 


\section{REFERENCES}

[1] M.Minnaert, Phil.Mag., 16, 235 (1933)

[2] T.G.Leighton and A.J.Walton, Eur.J.Phys., 8, 98 (1987)

[3] W.E.Farrell, D.P.McKenzie, and R.L.Parker, Proc.Camb.Phil.Soc., 65, 365 (1969)

[4] F.S.Crawford, Am.J.Phys., 50, no.5, 398 (1982)

[5] C.Devin, J.Acous.Soc.Am., 31, no.12, 1654 (1959)

[6] A.Prosperetti, J.Fluid Mech., 168, 457 (1986)

[7] A.Prosperetti, J.Fluid Mech., 222, 587 (1991)

[8] E.L.Carstensen and L.L.Foldy, J.Acous.Soc.Am., 19, no.3, 481 (1947)

[9] E.Silberman, J.Acous.Soc.Am., 29, no.8, 925 (1957)

[10] L.d'Agostino and C.E.Brennen, J.Acous.Soc.Am., 84, 2126 (1988)

[11] T.G.Leighton, "The Acoustic Bubble", Academic Press (London) 1994

[12] G.N.Kuznetsov and I.E.Shchekin, Akust.Zh.,18, 565 (1972) [Sov.Phys.Acoust., 18, 466 $(1973)]$

[13] Yu.A.Kobelev and L.A.Ostrovskii, Akust.Zh.,30,715 (1984) [Sov.Phys.Acoust., 30, 427 (1984)]

[14] V.V.Bredikhin, Yu.A.Kobelev, and N.I.Vasilinenko, J.Acoust.Soc.Am., 103, 1775 (1998)

[15] M.Strasberg, J.Acous.Soc.Am., 25, 536 (1953)

[16] P.H.Roberts and C.C.Wu, Phys.Fluids, 10, no.12, 3227 (1998) 
[17] M.Strasberg, J.Acous.Soc.Am., 28, 20 (1956)

[18] S.D.Howkins, J.Acous.Soc.Am., 37, 504 (1964)

[19] A.Prosperetti, J.Acous.Soc.Am., 61, 17 (1977) 


\section{FIGURE CAPTIONS}

FIG.1 Simple tool for capturing the bubble

FIG.2 Diagram of the experimental setup. In the experiment, we pump air into a tube immersed in water to produce the bubbles. The radii difference between these bubbles is small and will be neglected. (It can be shown that a small radii difference yields second order correction of $\omega_{s}$ and $\left.\omega_{a}\right)$.

FIG.3 Different geometrical configurations

FIG.4 (a) Spectra of symmetrical and antisymmetrical modes in configuration 3(a).

(b) Change of sign of $\operatorname{Im} p$ for the antisymmetrical mode when configuration 3(b) is adopted.

FIG.5 Linear fitting of the plot $\frac{1}{f_{s, a}^{2}} \cdot 10^{7}\left(\mathrm{~Hz}^{-2}\right)$ vs. $\frac{1}{d}\left(\mathrm{~cm}^{-1}\right)$ for the two modes. Average of the resonance frequency of the two bubbles: $1499 \mathrm{~Hz}$; corresponding radius: $0.217 \mathrm{~cm}$; slopes for the two fitting lines: 0.578 and $-0.550\left(\mathrm{~cm} \cdot \mathrm{sec}^{2}\right)$; slopes of theoretical prediction: $\pm 0.966\left(\mathrm{~cm} \cdot \mathrm{sec}^{2}\right)$. 


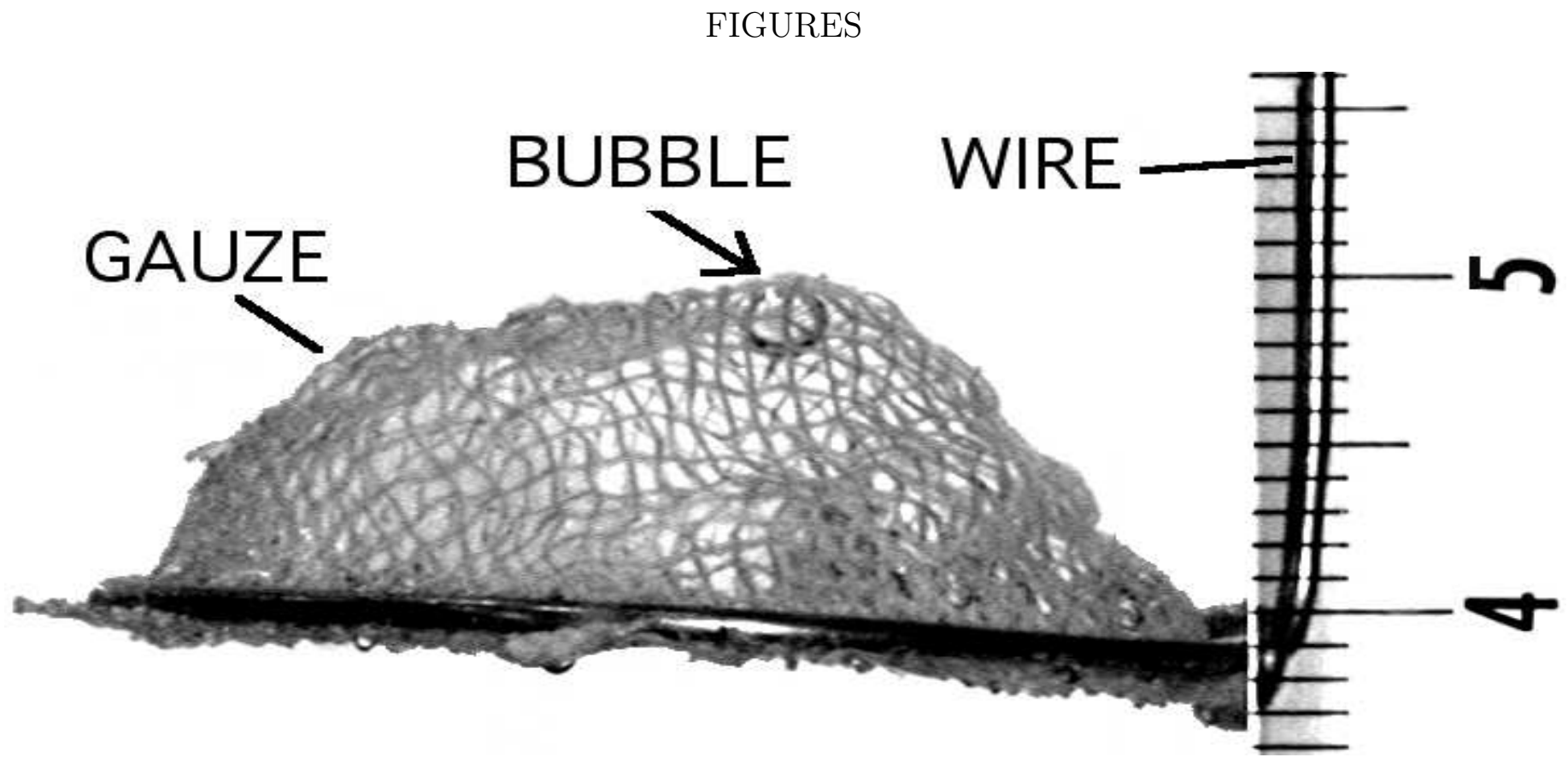

FIG. 1 


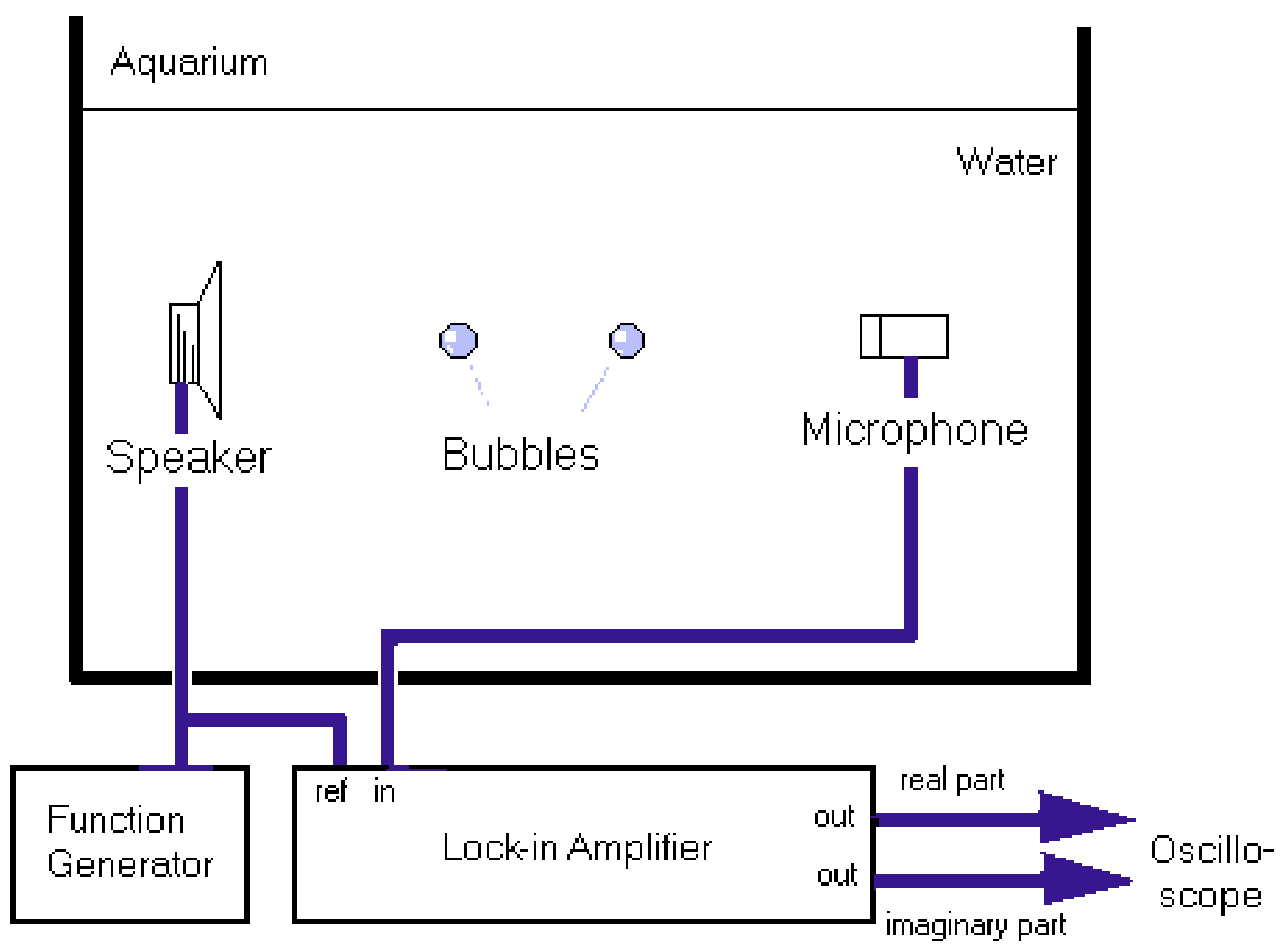

FIG. 2 


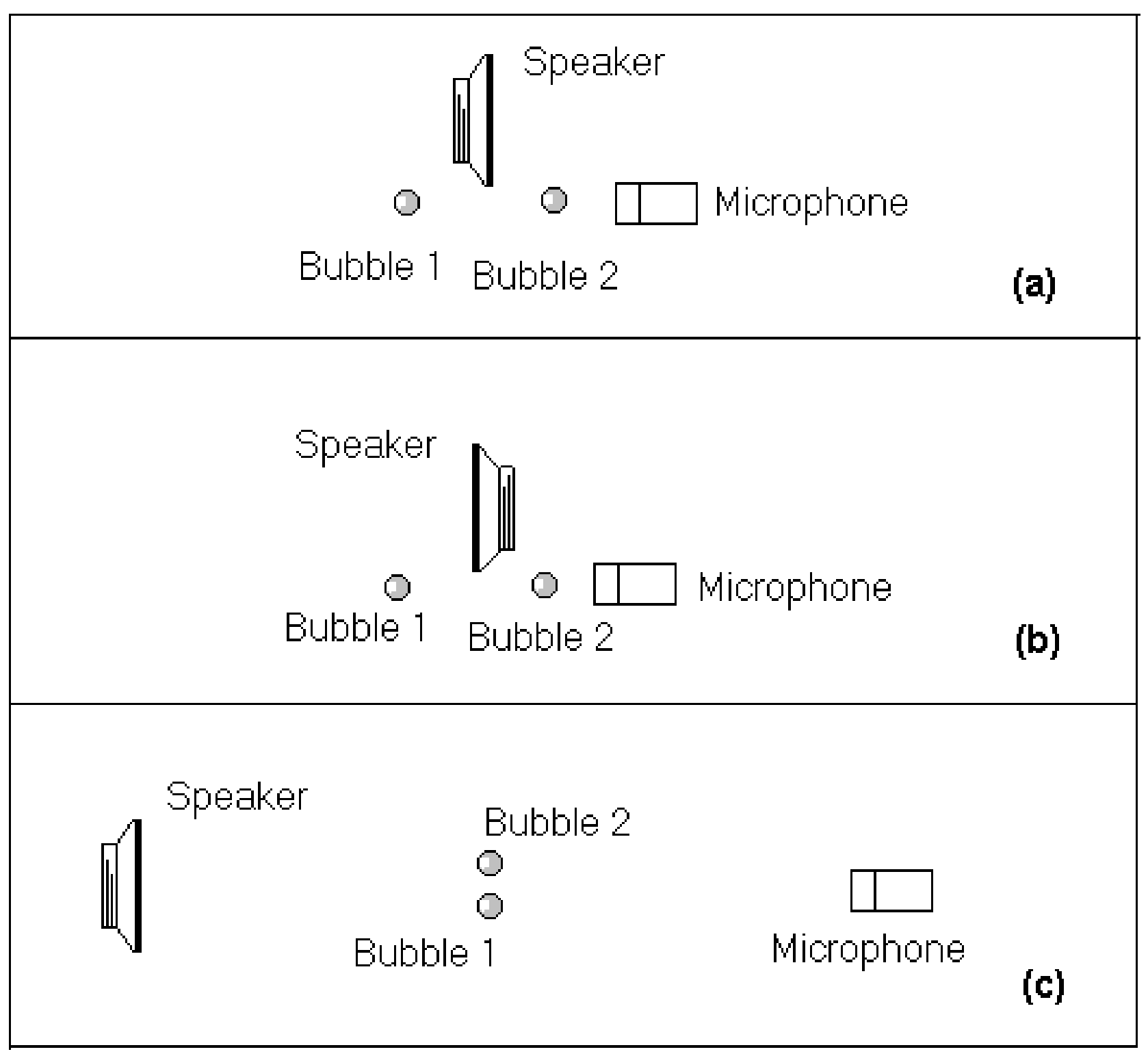

FIG. 3 


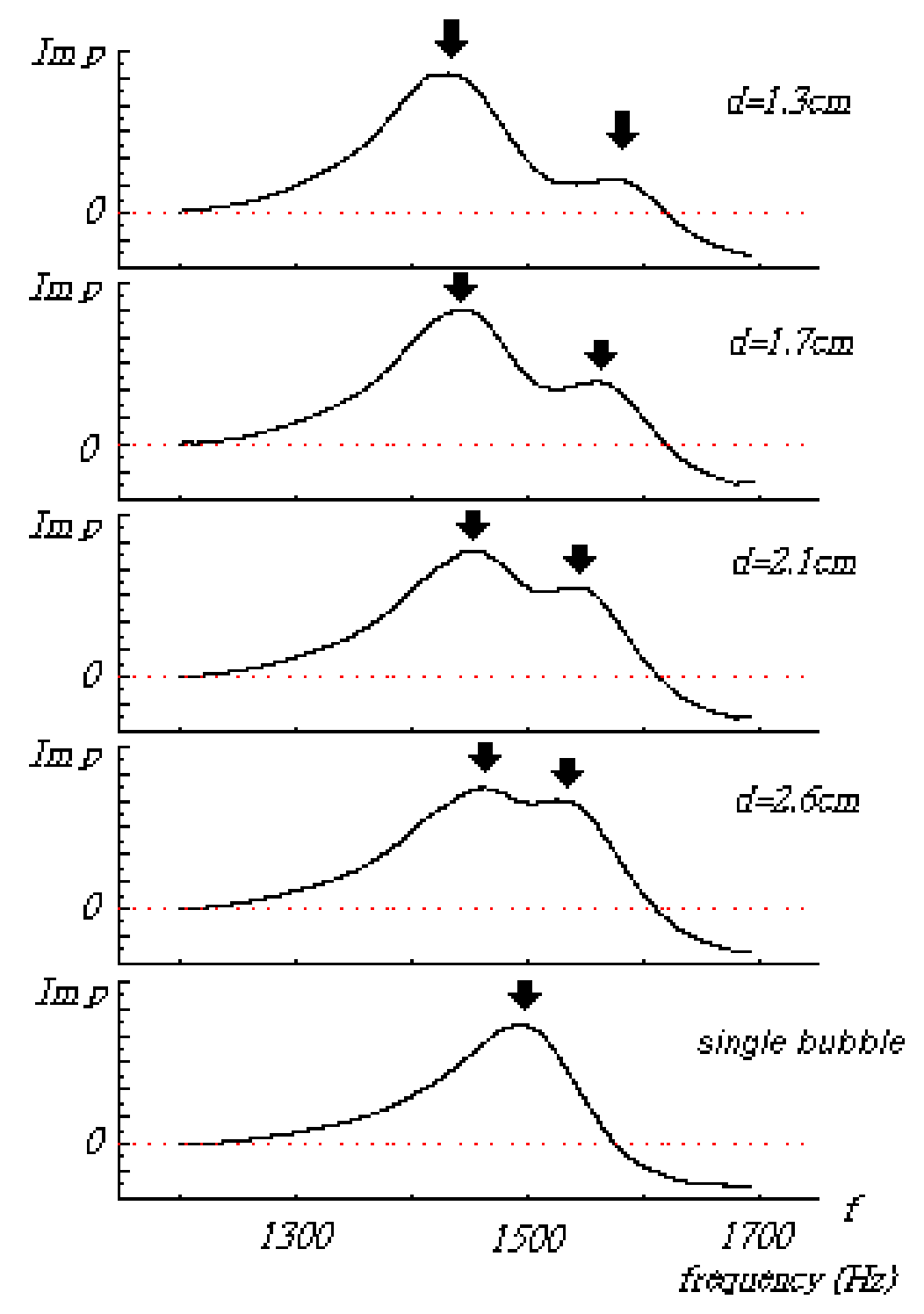

FIG. 4(a) 


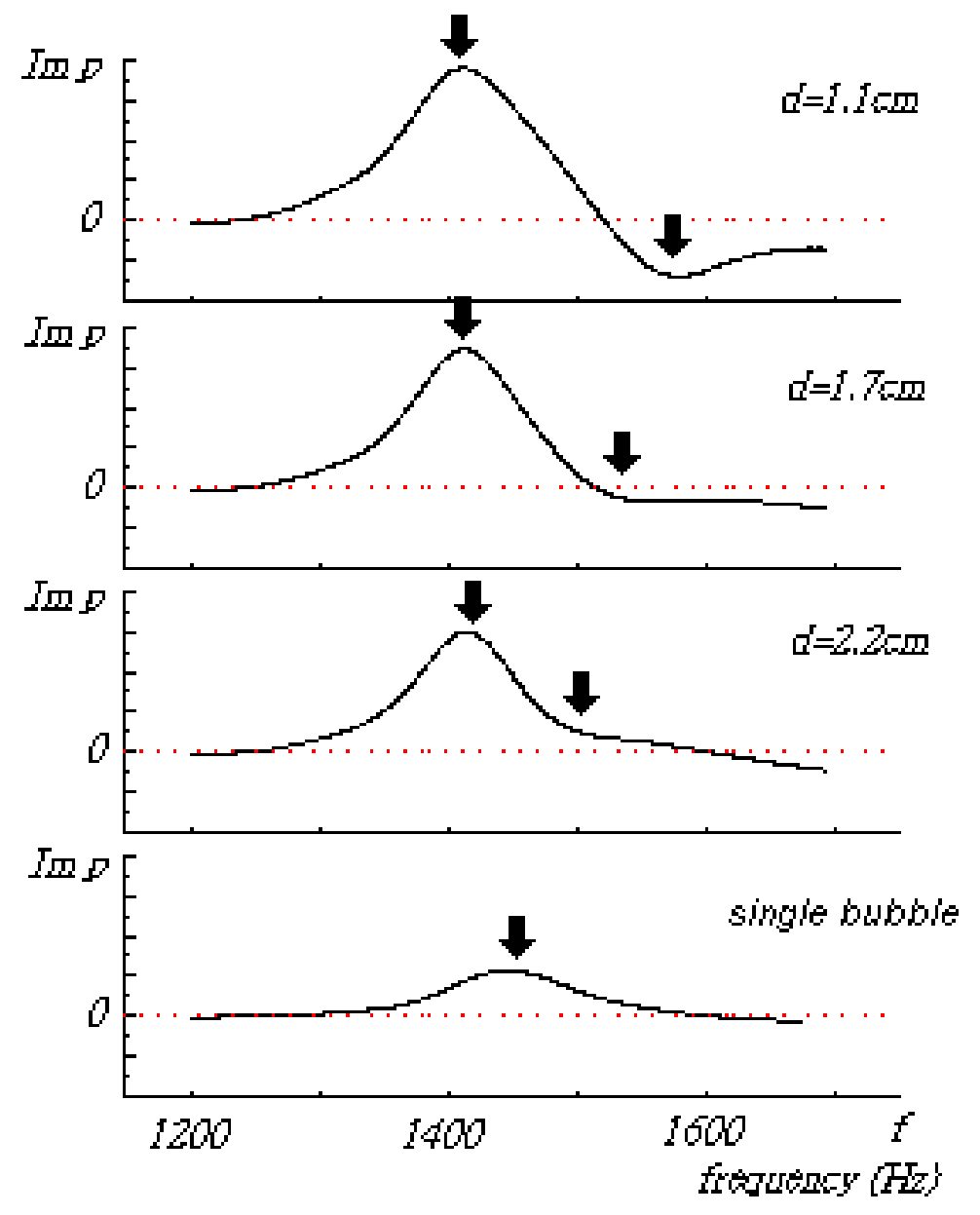

FIG. 4(b) 


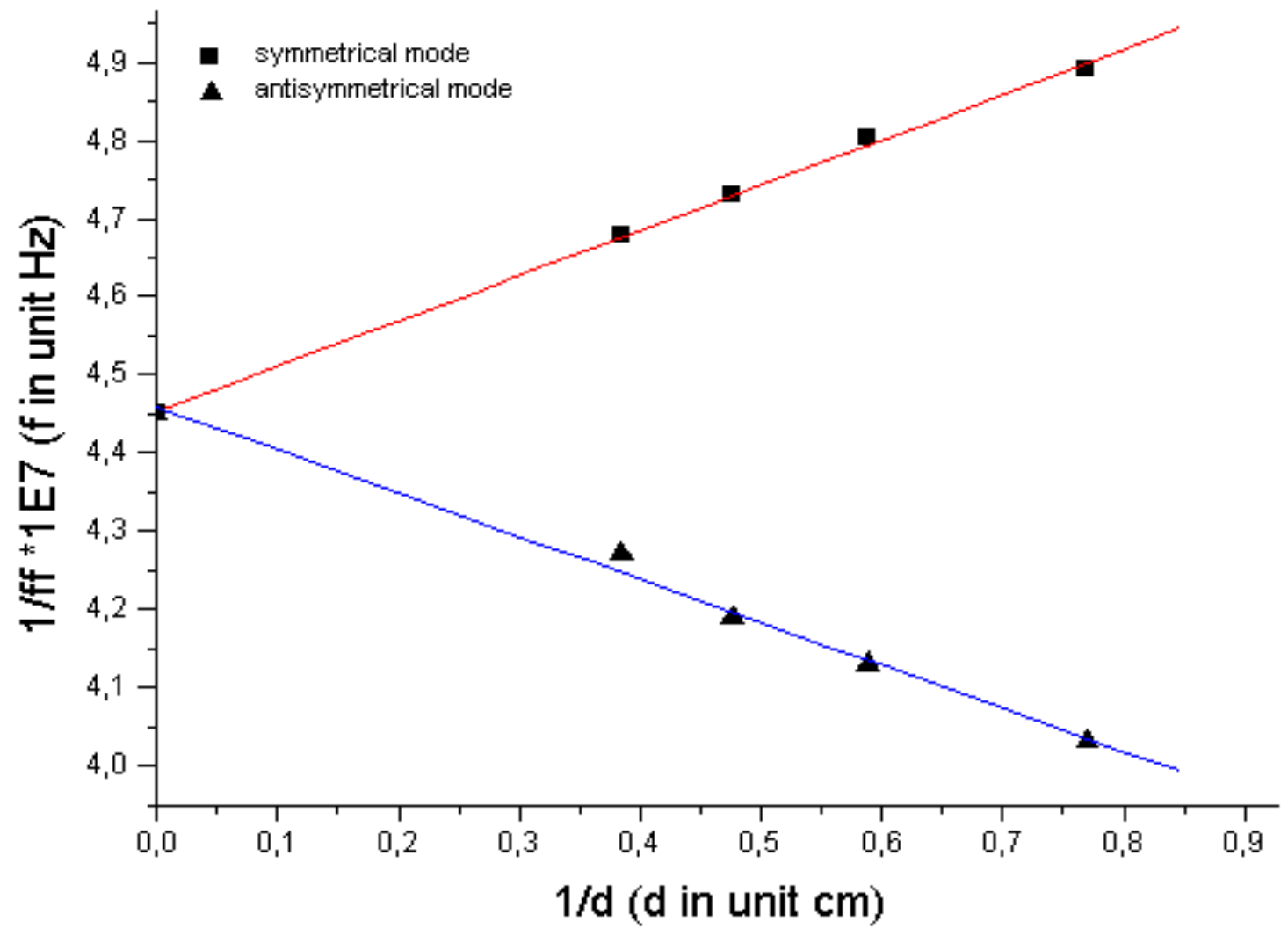

FIG. 5 


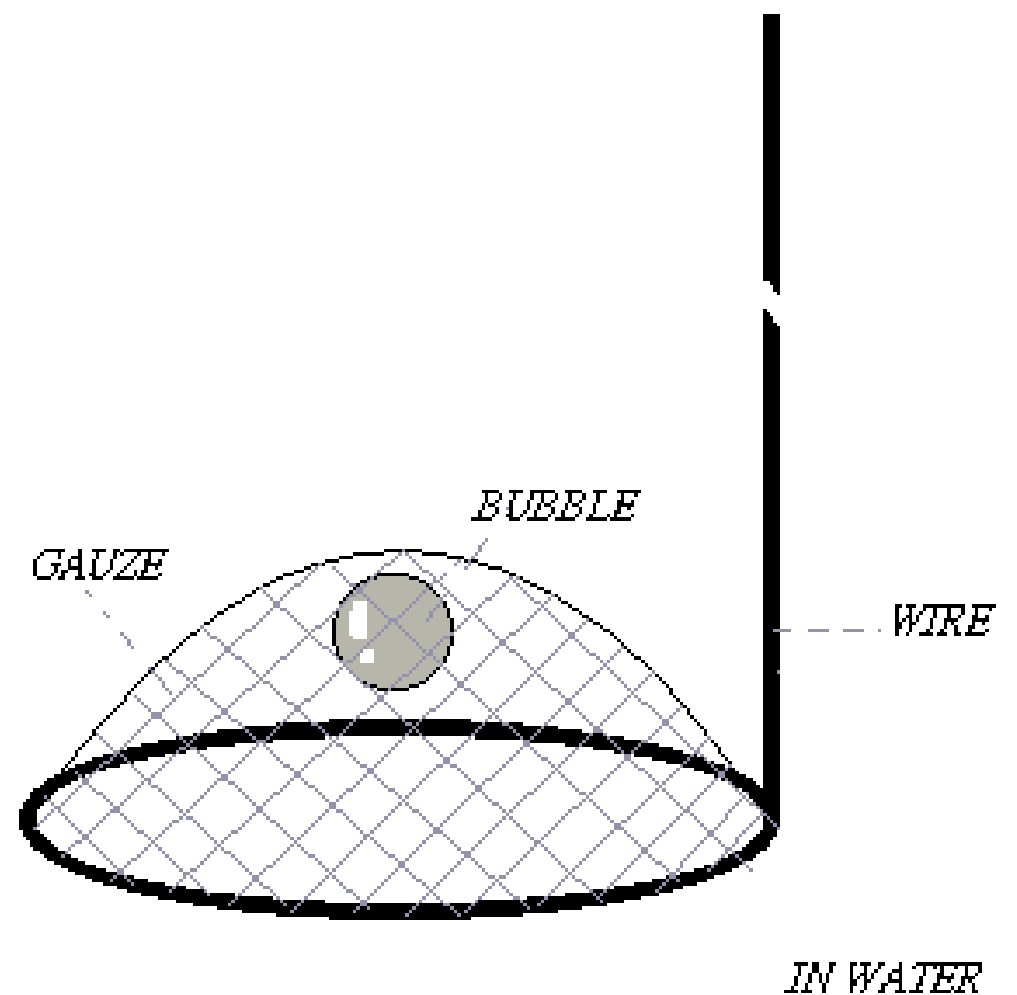

